\title{
Differences in Metabolic Network Modulation Between Capsulotomy and Deep-Brain Stimulation for Refractory Obsessive-Compulsive Disorder
}

\author{
Kristin Suetens*1, Bart Nuttin*2, Loes Gabriëls*3, and Koen Van Laere ${ }^{1}$ \\ ${ }^{I}$ Nuclear Medicine and Molecular Imaging, University Hospital and KU Leuven, Leuven, Belgium; ${ }^{2}$ Department of Neurosurgery, \\ University Hospital and KU Leuven, Leuven, Belgium; and ${ }^{3}$ Department of Psychiatry, University Hospital and KU Leuven, \\ Leuven, Belgium
}

Around $7 \%-10 \%$ of patients with obsessive-compulsive disorder (OCD) are refractory to first-line treatment. Neurosurgical approaches are available such as capsulotomy or deep-brain stimulation (DBS). There is strong evidence for central involvement of the corticostriatopallidothalamocortical (CSPTC) circuit in OCD, but the exact mechanism through which these interventions lead to clinical improvement and potential differences in network modulation are not fully understood. Methods: In total, 13 capsulotomy patients (aged 29-59 y, 10 men and 3 women) and 16 DBS patients (aged 25-56 y, 6 men and 10 women) were prospectively included. ${ }^{18} \mathrm{~F}$-FDG PET was performed before and after capsulotomy and before and after DBS in both stimulation-on and stimulation-off conditions. Presurgical scans were compared with scans of healthy volunteers using SPM8 and global scaling, and metabolic changes after DBS were compared with changes after capsulotomy. Correlations with clinical improvements were investigated using the Yale-Brown ObsessiveCompulsive Scale (Y-BOCS) and the Hamilton Depression Rating Scale (HAM-D). Results: Both groups had similar pretreatment clinical morbidity as assessed by Y-BOCS and the Hamilton Depression Rating Scale. Preoperative superior frontal and supplementary motor cortex hypometabolism was common to both patient groups, and the subgenual anterior cingulate, occipital cortex (cuneus), and posterior cerebellum were relatively hypermetabolic. Postoperative metabolic decreases were common to both interventions in the anterior cingulate and the prefrontal and orbitofrontal cortices. Compared with DBS, capsulotomy resulted in more intense metabolic changes, with additional significant decreases in the mediodorsal thalamus, caudate nucleus, and cerebellum as well as increases in the precuneus and the fusiform and lingual gyrus. The stimulation-off condition of DBS patients showed no significant differences from the preoperative state. Improvement in Y-BOCS scores correlated with metabolic changes in the occipital cortex. Baseline metabolism in the subgenual anterior cingulate and superior temporal cortices were related to postoperative improvement of depressive symptoms. Conclusion: Capsulotomy and DBS lead to similar clinical improvement and similar metabolic network changes in the CSPTC circuit, with a prominent role for the subgenual anterior cingulate and other core structures of the CSPTC. However, metabolic changes are more pronounced and extended in capsulotomy than in DBS. Furthermore, cortical regions outside the CSPTC may also play an important role in OCD symptomatology.

Received Jun. 3, 2013; revision accepted Jan. 1, 2014.

For correspondence or reprints contact: Koen Van Laere, University Hospital Leuven, Herestraat 49, E901, 3000 Leuven, Belgium.

E-mail: koen.vanlaere@uzleuven.be

${ }^{*}$ Contributed equally to this work.

Published online Apr. 10, 2014.

COPYRIGHT (C) 2014 by the Society of Nuclear Medicine and Molecular Imaging, Inc.
Key Words: obsessive-compulsive disorder; positron emission tomography; deep-brain stimulation; anterior capsulotomy; anterior cingulate; Y-BOCS

J Nucl Med 2014; 55:951-959

DOI: $10.2967 /$ jnumed.113.126409

$\mathbf{O}$ bsessive-compulsive disorder (OCD) is an anxiety disorder with obsessions or compulsions as the core symptoms. It is a relatively common disorder with an estimated lifetime prevalence of $2 \%-3 \%$ worldwide (1). The disorder is often accompanied by other anxiety disorders and major depression. First-line treatment generally consists of cognitive-behavioral or pharmacologic therapy with selective serotonin reuptake inhibitors. A minority of about $7 \%-$ $10 \%$ of the patients becomes refractory to all available first-line treatments. For the most severe refractory cases, neurosurgical interventions have been developed since the 1960s. Initially, ablative approaches were applied, such as capsulotomy, subcaudate tractotomy, anterior cingulotomy, and limbic leucotomy (2). In 1999, deep-brain stimulation (DBS) of the anterior limb of the internal capsule and the gray matter just ventral to the anterior limb of the internal capsule was introduced (3). Benefits of DBS are the theoretic reversibility of the intervention and patient-specific adjustability of stimulation parameters. In general, a neurosurgical intervention is effective, with an OCD-symptom response rate of about $50 \%-60 \%$, and comorbid depressive symptoms often are alleviated as well (4).

The exact mechanism through which electrical capsular stimulation and capsulotomy lead to improvement of symptom severity is not completely known. There is strong evidence for central involvement of the corticostriatopallidothalamocortical (CSPTC) circuit in OCD, especially the limbic loop including the medial orbitofrontal cortex and the anterior cingulate projecting to the mediodorsal thalamus (4-6).

Previous neuroimaging studies have found changes in metabolism in crucial CSPTC structures in both untreated and treated OCD patients. In untreated OCD patients without comorbidity, hypermetabolism in parts of the frontal cortex, caudate nuclei, anterior cingulate gyrus, and thalamus are most consistently reported (7-9).

Regarding the functional alterations on capsular DBS for OCD, Rauch et al. found activation in parts of the frontal cortex, anterior cingulate, striatum, pallidum, and thalamus in a study on 6 patients after acute stimulation (10). Our group found decreased prefrontal metabolic activity after DBS in 6 patients, in whom decreases on 
the Yale-Brown Obsessive-Compulsive Scale (Y-BOCS) and Hamilton Depression Rating Scale (HAM-D) were inversely correlated to changes in left ventral striatum, left amygdala, and left hippocampus metabolic activity (11) and preoperative metabolism in the subgenual anterior cingulate was predictive of therapeutic response. Similarly, Le Jeune et al. (8) found that subthalamic stimulation led to a decrease in prefrontal and anterior cingulate metabolism. Recently, Zuo et al. assessed glucose metabolic changes in 8 patients after capsulotomy and also reported decreased metabolism in the prefrontal cortex and anterior cingulate cortex (ACC) but increases in the precentral and lingual gyri. Clinical improvement correlated with metabolic changes in the right and left dorsal ACC and in the right middle occipital gyrus (12).

The goal of this study was 4-fold: first, to compare the baseline metabolism of patients selected for capsulotomy versus chronic DBS relative to healthy volunteers, in relation to their symptomatology; second, to compare the functional metabolic changes after capsulotomy with chronic DBS on a voxelwise basis; third, to investigate metabolic alterations in the DBS-on and DBS-off conditions and assess whether the DBS-off condition is distinguishable from the baseline preoperative situation; and fourth, to explore the relationship between treatment outcome regarding OCD and depression symptomatology versus the observed metabolic changes.

\section{MATERIALS AND METHODS}

\section{Participants}

Two groups of treatment-refractory OCD patients were prospectively included in this study. The first group (capsulotomy group) consisted of 13 patients who underwent an anterior capsulotomy between 2001 and 2009 (aged 29-59 y; mean age, $39.0 \pm 8.9$ y; 10 men and 3 women). Detailed demographics and symptomatology are given in Tables 1 and 2. The second group (DBS group) consisted of 16 patients (aged 25-56 y; mean age, $39.0 \pm 9.5 \mathrm{y} ; 6$ men and 10 women) who were treated with DBS between 1998 and 2009. The 6 DBS patients reported previously by Van Laere et al. (11) were also included in this group.

To be qualified to undergo a neurosurgical intervention, the patients had to be diagnosed with severe, disabling OCD as defined by the Diagnostic and Statistical Manual of Mental Disorders, version IV
(13). All patients had a score of 45 or less on the Global Assessment of Functioning Scale, for a minimum of $5 \mathrm{y}$. Their inclusion Y-BOCS score was 30 or above. Furthermore, all patients had to meet strict criteria for treatment refractoriness, defined as ongoing failure of at least 3 trials with selective serotonin reuptake inhibitors, of which clomipramine was one, and augmentation therapy with at least 2 atypical antipsychotics and adequate cognitive behavioral therapy while on a therapeutic dose of a selective serotonin reuptake inhibitor (14). At inclusion, the patient's age had to be between 18 and $65 \mathrm{y}$ (14).

Exclusion criteria included any history of current or past psychotic disorder, a manic episode in the past $3 \mathrm{y}$, current clinically significant neurologic disorders or medical illnesses affecting brain function or structure (except for tic disorder), and current or unstably remitted substance abuse or dependence. OCD patients with a comorbid diagnosis of cluster $\mathrm{B}$ personality disorder and a history of frequent acting-out behavior or noncompliance with treatment were excluded (14).

For DBS, the inclusion criteria were stricter, as possible DBS candidates had to comply with the demanding protocol and frequent follow-up evaluations. The candidates had to participate in a double-masked randomized trial with stimulation turned on or off. Patients approved for DBS could also opt for capsulotomy (14).

All included patients underwent surgical treatment by the same neurosurgeon and were evaluated by an experienced psychiatrist. The DBS parameters were individually optimized after surgery. During the masked cross-over, the optimized stimulation parameters remained stable. At the time of clinical evaluation of the DBS patients, both the evaluator and the patient were masked to treatment status. The medication scheme of all patients was carefully monitored during the whole period, and medication was reduced as low as possible before the first PET scan was taken. During the entire imaging study, medication was kept stable.

To estimate OCD symptom severity, Y-BOCS was assessed (15). Depression symptomatology was measured by the 17-item Hamilton Depression Rating Scale (HAM-D) (16). Figure 1 shows preoperative and postoperative individual and average obsessive-compulsive and depressive symptomatology for both groups. The clinical scores used in PET data analysis are those obtained closest to the date of the PET scan concerned. The PET scan during the stimulation-off condition was available for only 9 patients ( 3 men and 6 women) in the DBS group. When stimulation was off, the OCD symptoms worsened considerably

TABLE 1

Demographics and Symptomatology for CAPS Patients

\begin{tabular}{|c|c|c|c|c|c|c|c|c|}
\hline \multirow[b]{2}{*}{ Patient no. } & \multirow[b]{2}{*}{ Age $(\mathrm{y})$ at intervention } & \multirow[b]{2}{*}{ Sex } & \multicolumn{3}{|c|}{ Y-BOCS } & \multicolumn{3}{|c|}{ HAM-D } \\
\hline & & & Preop & Postop & Change (\%) & Preop & Postop & Change (\%) \\
\hline 1 & 34 & $M$ & 32 & 18 & 43.8 & 16 & 14 & 12.5 \\
\hline 2 & 46 & M & 32 & 24 & 25.0 & 20 & 12 & 40.0 \\
\hline 3 & 59 & M & 32 & 12 & 62.5 & 28 & 7 & 75.0 \\
\hline 4 & 45 & M & 33 & 22 & 33.3 & 18 & 14 & 22.2 \\
\hline 5 & 40 & M & 30 & 0 & 100.0 & 21 & 2 & 90.5 \\
\hline 6 & 43 & M & 37 & 16 & 56.8 & 20 & 12 & 40.0 \\
\hline 7 & 31 & M & 26 & 9 & 65.4 & 26 & 7 & 73.1 \\
\hline 8 & 41 & M & 32 & 23 & 28.1 & 27 & 20 & 25.9 \\
\hline 9 & 27 & $M$ & 30 & 27 & 10.0 & 15 & 8 & 46.7 \\
\hline 10 & 33 & $\mathrm{~F}$ & 28 & 18 & 35.7 & 18 & 15 & 16.7 \\
\hline 11 & 28 & $\mathrm{~F}$ & 31 & 20 & 35.5 & 18 & 8 & 55.6 \\
\hline 12 & 36 & $\mathrm{~F}$ & 38 & 7 & 81.6 & 11 & 11 & 0.0 \\
\hline 13 & 45 & $M$ & 36 & 7 & 80.6 & 24 & 4 & 83.3 \\
\hline Mean \pm SD & $39.1 \pm 8.9$ & & $32.1 \pm 3.4$ & $15.6 \pm 8.0$ & $50.6 \pm 26.3$ & $20.2 \pm 5.0$ & $10.3 \pm 4.9$ & $44.7 \pm 29.1$ \\
\hline
\end{tabular}

Scores are those obtained closest to the date of the PET scan concerned. 
TABLE 2

Demographics and Symptomatology for DBS Patients

\begin{tabular}{|c|c|c|c|c|c|c|c|c|}
\hline \multirow[b]{2}{*}{ Patient no. } & \multirow[b]{2}{*}{ Age $(y)$ at intervention } & \multirow[b]{2}{*}{ Sex } & \multicolumn{3}{|c|}{ Y-BOCS } & \multicolumn{3}{|c|}{ HAM-D } \\
\hline & & & Preop & Postop (DBS on) & Change (\%) & Preop & Postop (DBS on) & Change (\%) \\
\hline 1 & 38 & $\mathrm{~F}$ & 30 & 21 & 30.0 & 29 & 26 & 10.3 \\
\hline 2 & 26 & $\mathrm{~F}$ & 31 & 13 & 58.1 & 25 & 7 & 72.0 \\
\hline 3 & 31 & $\mathrm{M}$ & 32 & 17 & 46.9 & 21 & 4 & 81.0 \\
\hline 4 & 39 & $\mathrm{~F}$ & 34 & 25 & 26.5 & 19 & 10 & 47.4 \\
\hline 5 & 25 & M & 33 & 25 & 24.2 & 21 & 7 & 66.7 \\
\hline 6 & 49 & $\mathrm{~F}$ & 33 & 36 & -9.1 & 18 & 26 & 44.4 \\
\hline 7 & 39 & $\mathrm{~F}$ & 35 & 10 & 71.4 & 16 & 6 & 62.5 \\
\hline 8 & 51 & $\mathrm{~F}$ & 33 & 20 & 39.4 & 23 & 17 & 26.1 \\
\hline 9 & 51 & $\mathrm{~F}$ & 37 & 30 & 18.9 & 24 & 12 & 50.0 \\
\hline 10 & 33 & M & 37 & 22 & 40.5 & 29 & 6 & 79.3 \\
\hline 11 & 34 & M & 38 & 30 & 21.1 & 43 & 28 & 34.9 \\
\hline 12 & 56 & $\mathrm{~F}$ & 36 & 5 & 86.1 & 26 & 4 & 84.6 \\
\hline 13 & 29 & $\mathrm{~F}$ & 37 & 2 & 94.6 & 21 & 5 & 76.2 \\
\hline 14 & 47 & $\mathrm{~F}$ & 34 & 21 & 38.2 & 6 & 5 & 16.7 \\
\hline 15 & 37 & M & 30 & 12 & 60.0 & 24 & 11 & 54.2 \\
\hline 16 & 42 & M & 32 & 19 & 40.6 & 25 & 8 & 68.0 \\
\hline Mean \pm SD & $39.2 \pm 9.5$ & & $33.9 \pm 2.6$ & $19.3 \pm 9.2$ & $43.0 \pm 26.4$ & $23.1 \pm 7.7$ & $11.4 \pm 8.3$ & $49.1 \pm 34.0$ \\
\hline
\end{tabular}

Scores are those obtained closest to the date of the PET scan concerned.

in most patients. The research protocol allowed us to proceed to the next phase of the study (i.e., go from on to off or from off to on or finish the cross-over) if the patient deteriorated clinically to an intolerable level, as evaluated by the patient and the psychiatrist, both remaining masked to the stimulation condition. Most stimulation-off conditions appeared to be shortened, and several PET scans could not be rescheduled within the shortened time frame for the stimulation-off condition.

Preoperative patient scans were compared with those of a historical group of 26 screened healthy volunteers ( 13 men and 13 women) with a mean age $\pm \mathrm{SD}$ of $32 \pm 8.9 \mathrm{y}$ (range, $21-47 \mathrm{y}$ ), under the same acquisition and reconstruction protocol using the same scanner.

All subjects gave written informed consent to participate in the study, which was approved by the local University Ethics Committee.

\section{Surgical Procedure}

The surgical procedure has been described in detail elsewhere $(4,17)$. Briefly, the radiofrequency capsulotomy lesions were $6 \mathrm{~mm}$ anterior to the posterior border of the anterior commissure, with a precoronal entry point, and were in the internal capsule and the gray matter just ventral to the internal capsule. A lesion was produced by heating the tip of a 10-mm-long electrode (Radionics) to $80^{\circ} \mathrm{C}$ during $2 \mathrm{~min}$. Afterward, the electrode was retracted $10 \mathrm{~mm}$ and a second lesion was made. This procedure was performed bilaterally. For DBS, bilateral electrodes and stimulators were implanted as described previously (17).

\section{PET Procedure}

All patients underwent preoperative and postoperative ${ }^{18} \mathrm{~F}-\mathrm{FDG}$ PET. For the DBS group, 9 of the 16 patients were also scanned in the stimulation-off condition (stimulation-on and stimulation-off scans were obtained in randomized order, and the PET evaluator was masked to the condition).

The preoperative scan was acquired an average of $28 \pm 41 \mathrm{~d}$ (range, $1-152 \mathrm{~d}$ ) before the surgical intervention in the capsulotomy group and $43 \pm 51 \mathrm{~d}$ (range, 2-148 d) in the DBS group. The capsulotomy group was scanned postoperatively an average of $107 \pm 43 \mathrm{~d}$ (range, 5-190 d) after surgical treatment.

The mean time between clinical scoring and preoperative PET was $27 \pm 11 \mathrm{~d}$ (range, 7-55 d) in the capsulotomy group and $21 \pm 9 \mathrm{~d}$ (range,
5-42 d) in the DBS group. The mean time between scoring and the postoperative scan was $26 \pm 6 \mathrm{~d}$ (range, 1-32 d) for the capsulotomy group.

During the DBS-on condition, the patients underwent postoperative PET after continuous electrical stimulation for a median of $68 \mathrm{~d}$ (range, 28-123 d). During the DBS-off condition, the patients underwent postoperative PET after a median of $24 \mathrm{~d}$ (range, 7-99 d) without electrical stimulation. The median time between scoring and the postoperative scan was $13 \mathrm{~d}$ (range, 4-42 d) during the DBS-on condition and $12 \mathrm{~d}$ (range, 0-62 d) during the DBS-off condition.

PET scans were acquired using an ECAT HR + camera (Siemens) operated in 3-dimensional mode. ${ }^{18} \mathrm{~F}-\mathrm{FDG}(150-185 \mathrm{MBq})$ was injected intravenously under standard conditions, that is, with the patients supine with ears and eyes open in a dimly lit, quiet room. Thirty minutes after ${ }^{18}$ F-FDG injection, a 30 -min dynamic scan $(6 \times 5$ min) began. During the acquisition, the patient's head was immobilized by a vacuum pillow. Attenuation was corrected using a ${ }^{68} \mathrm{Ge} /{ }^{68} \mathrm{Ga}$ transmission scan,

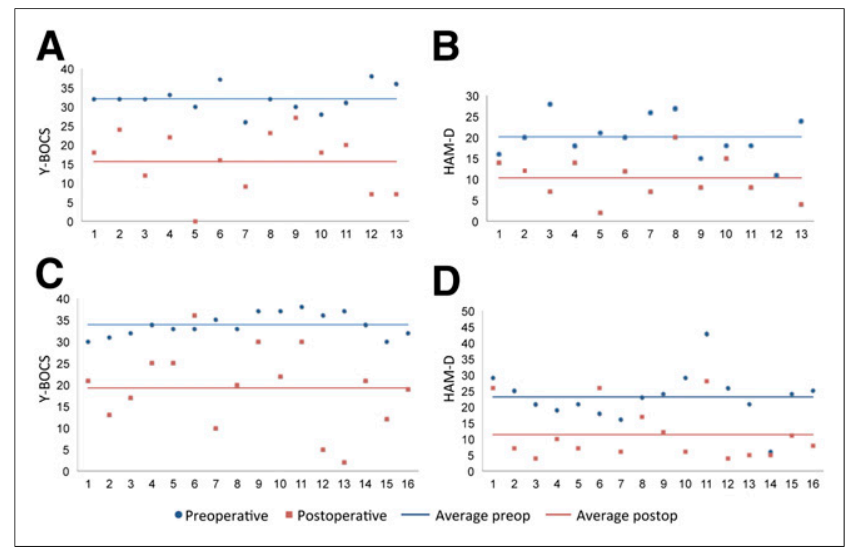

FIGURE 1. Preoperative and postoperative individual and average clinical scores. (A and B) Capsulotomy group. (C and D) DBS group. Clinical scores are those obtained closest to the date of the PET scan concerned. 
scatter was corrected, and the images were reconstructed using 3-dimensional filtered backprojection with a Hanning postprocessing filter of $6 \mathrm{~mm}$ in full width at half maximum.

\section{Image Analysis}

The data were analyzed using statistical parametric mapping (SPM8; Wellcome Trust Centre for Neuroimaging) for PET/SPECT (basic models), implemented in MATLAB 7 (The MathWorks).

Patient scans were corrected for motion using individual frames. The postoperative scans were coregistered to the preoperative scan for each patient. Next, the mean image of these coregistered images was calculated and spatially normalized to Montreal Neurologic Institute (MNI) space using the SPM8 PET template and nonrigid registration with 16 iterations and gaussian smoothing of $8 \mathrm{~mm}$ in full width at half maximum. The resulting voxels were $2 \times 2 \times 2 \mathrm{~mm}$. The geometric transformation obtained by this spatial normalization of the mean image was also applied to the preoperative and postoperative images.

Gray matter was segmented using the SPM8 gray matter probability map with a threshold of 0.27 , which was a visual compromise between gray matter preservation and white matter exclusion and robust within the range of $0.25-0.30$. After the average gray matter activity of each image had been normalized, the preoperative image was subtracted from the postoperative image, from which $z$ score parametric maps were calculated.

A 2-sample $t$ test was used to compare the preoperative resting metabolism of the capsulotomy and DBS groups with the healthy group. One-sample $t$ tests were used to find significant postoperative changes in the capsulotomy and DBS groups using the $z$ score maps. A 1-sample $t$ test was also used to compare the DBS-on versus DBS-off condition $(n=9)$ by replacing the preoperative images by the DBSoff images to calculate the $z$ scores.

A conjunction analysis was performed to discover the postoperative changes that are jointly significant in the capsulotomy and the complete DBS groups $(n=16)(18)$. For this analysis, we used the conjunction option in SPM8 with the contrasts $(0,-1)$ and $(-1,0)$ on the subtraction images. Subsequently, the postoperative metabolic changes in these 2 groups were compared using a 2-sample $t$ test.

Correlation analyses of $z$ score maps and baseline metabolism with changes in covariates Y-BOCS and HAM-D were performed. Age and sex were used as nuisance variables.

Unless specified otherwise, tests were performed with an uncorrected $P_{\text {height }}$ threshold of less than 0.001 and false-discovery rate (FDR)-corrected $P_{\text {cluster }}$ threshold of less than 0.05 , and with an extent threshold of 0 . To label the anatomic regions, we used the Talairach atlas (19). All data are displayed according to neurologic convention.

\section{RESULTS}

\section{Treatment Response}

Tables 1 and 2 give the response of patients to treatment. Improvements in obsessive-compulsive and depressive symptomatology are shown in Figure 1. When the capsulotomy and DBS groups were compared, the preoperative Y-BOCS and HAM-D scores were not significantly different. The decreases in the scores of the capsulotomy group as compared with those of the DBS-on group were also not significantly different.

Compared with preoperatively, Y-BOCS scores decreased significantly in the capsulotomy group $\left(16.5 \pm 9.1, P=10^{-5}\right)$ and in the DBS-on group $\left(14.6 \pm 9.5, P=10^{-5}\right)$. For the DBS group, the decrease in scores when stimulation was off compared with on was $5.8 \pm 6.9$, which was also significant $(P=0.02)$.

HAM-D scores decreased significantly in the capsulotomy group $\left(9.8 \pm 7.4, P=2.10^{-4}\right)$ and in the DBS-on group (11.7 $\left.\pm 8.1, P=2.10^{-5}\right)$. The DBS-off group showed a decrease of
$4.1 \pm 11.2$, compared with the preoperative condition-a difference that was not significant $(P=0.15)$.

\section{Baseline Characterization of Capsulotomy and DBS Groups}

Figure 2 shows the areas of significant changes in preoperative resting metabolism for the capsulotomy $(n=13)$ and DBS $(n=16)$ groups, compared with the healthy control group $(n=26)$. The cluster coordinates and statistics are given in Table 3. For both the capsulotomy group and the DBS group, clusters with relatively reduced preoperative resting metabolism were in the frontal cortex (right and left superior frontal gyrus, left supplementary motor cortex). We also found reduced preoperative resting metabolism in the right posterior cingulate cortex for the capsulotomy group and in the right parietal and right and left temporal cortices for the DBS group.

The inverse contrast showed relative increases in the occipital lobe, the ACC, and parts of the posterior cerebellum in both groups.

\section{Group and Conjunction Analysis of Postoperative Metabolic Changes}

Capsulotomy. Compared with baseline, there was decreased metabolic activity in the left dorsal ACC; the medial dorsal thalamus; the caudate head and body, extending toward the left lentiform nucleus and in the left subgenual ACC; bilaterally in the medial frontal cortex, extending toward the right insula and the right inferior frontal cortex; and bilaterally in the posterior cerebellum (pyramis). Relatively increased metabolic activity compared with baseline was found in the right occipital lobe and bilaterally in the

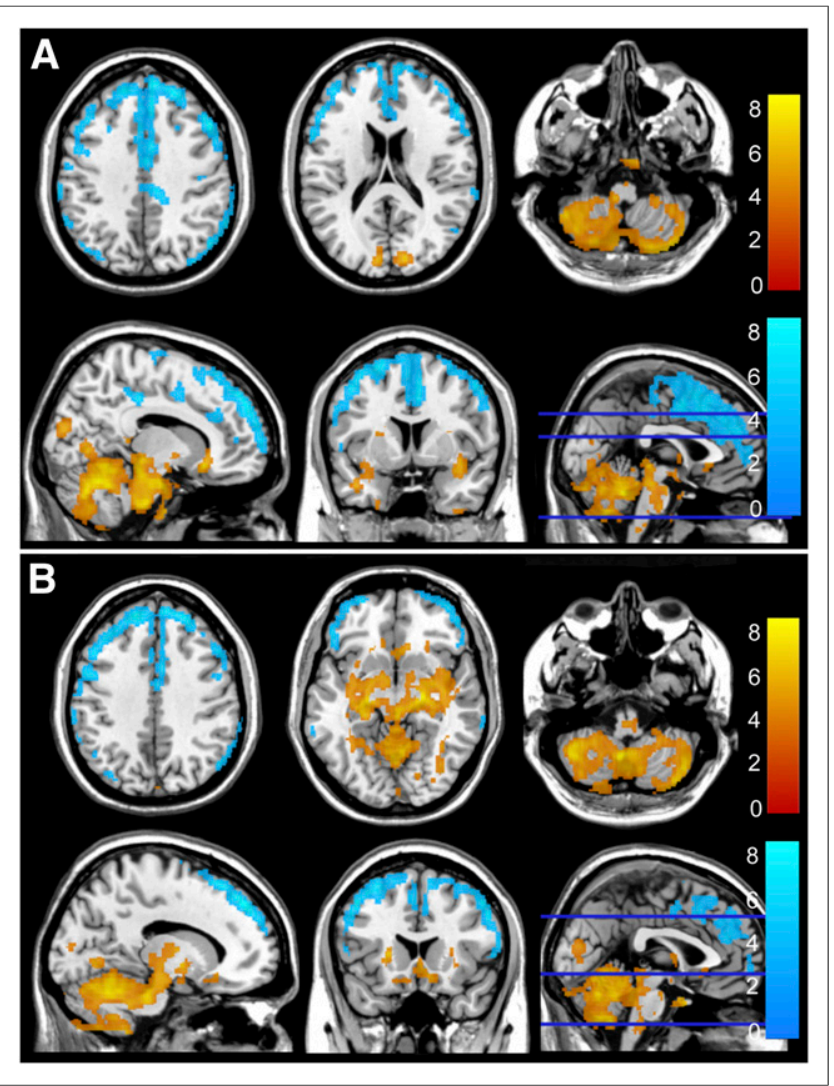

FIGURE 2. Areas of increased (red) and reduced (blue) preoperative resting metabolism compared with healthy control group for capsulotomy (A) and DBS (B) groups. 
TABLE 3

Global Cluster Peak Coordinates (Talairach) and Statistics of Postoperative Resting Metabolism Compared with Healthy Group

\begin{tabular}{cclrrr}
\hline Resting metabolism & $x, y, z(\mathrm{~mm})$ & \multicolumn{1}{c}{ Brain area } & $\begin{array}{c}P_{\text {height, }}, \\
\text { uncorrected }\end{array}$ & $\begin{array}{c}\text { Cluster } \\
\text { extent }\end{array}$ & $\begin{array}{c}P_{\text {cluster }} \\
\text { FDR-corrected }\end{array}$ \\
$t$ value
\end{tabular}

parietal lobe (postcentral gyrus), extending toward the precuneus. Figure $3 \mathrm{~A}$ shows the main significant clusters, and the statistics are given in Table 4.

$D B S$. In the DBS-on condition $(n=16)$, there was a significant relative metabolic decrease in the right dorsal ACC and in the frontal cortex (right middle and inferior, left superior and inferior) relative to the preoperative scan (Fig. 3B and Table 4 show statistics). In the DBS-off condition ( $n=9)$, no significant changes from the preoperative scan were seen, unless the significance levels were relaxed (uncorrected $P_{\text {height }}<0.005$, FDR-corrected $P_{\text {cluster }}>0.05$, and extent threshold $>100$ ). In that case, several regions showed reduced postoperative metabolism in the DBS-off condition, such as the right caudate nucleus and the medial dorsal thalamus.

Comparing the DBS-on and -off conditions of the 9 patients who underwent scanning under both conditions, significant decreases were present in the DBS-on condition in the ACC, the medial frontal gyrus, and the right temporal gyrus (data not shown).

Conjunction Analysis. A conjunction analysis identified the regions of postoperative metabolic changes that were jointly significant in the capsulotomy group $(n=13)$ and the complete DBS group $(n=16)$ (Fig. 4; Table 5). Similar to the group analysis, a cluster comprising the medial frontal cortex and inferior frontal cortex, extending toward the ACC and the caudate nucleus, showed common relative hypometabolism. In the medial dorsal thalamus there was a common hypometabolism in both groups when we relaxed our levels (uncorrected $P_{\text {height }}<0.001$ and FDR-corrected $P_{\text {cluster }}=0.10$ ). The conjunction tests did not show significant postoperative increases at the used thresholds.

\section{Metabolic Changes in Capsulotomy Versus DBS}

Figure 5 shows a significantly larger decrease in activity in the capsulotomy group $(n=13)$ than in the complete DBS group $(n=16)$ for the medial dorsal thalamus and the caudate heads. The statistics of these clusters are given in Table 6. No other significant differences in metabolic activity changes were found between the 2 groups.

\section{Relationship to Clinical Scores}

The analysis of correlations between relative (postoperative to preoperative) metabolism change and change in Y-BOCS score for both complete groups did not reveal any significant positive correlations at the cluster level. A significant negative correlation between metabolism change and change in Y-BOCS score was found in the capsulotomy group bilaterally in the occipital cortex

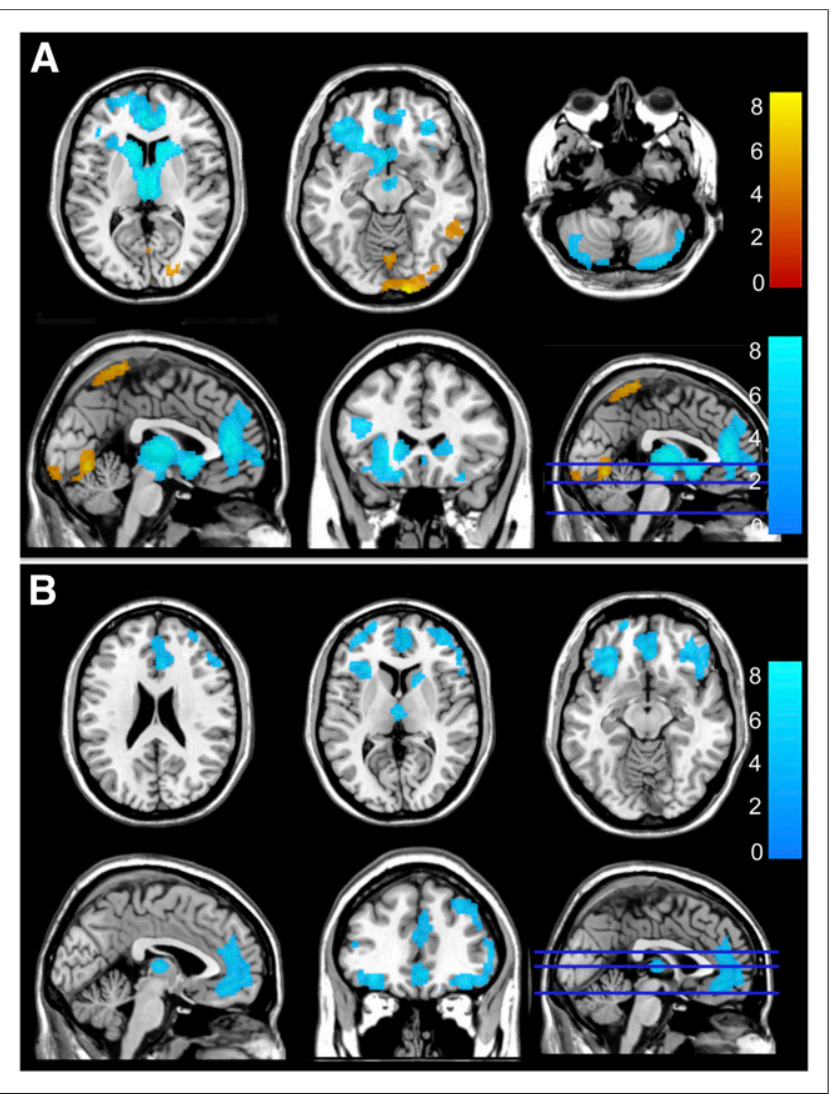

FIGURE 3. Significant postoperative changes in metabolic activity compared with baseline. (A) Capsulotomy group, showing decreases (blue) and increases (red). (B) DBS group in DBS-on condition, showing decreases only (no significant increases). 
TABLE 4

Coordinates and Statistics of Significant Postoperative Changes in Metabolic Activity

\begin{tabular}{|c|c|c|c|c|c|c|}
\hline Metabolic activity & $x, y, z(\mathrm{~mm})$ & Brain area & $\begin{array}{c}P_{\text {height }}, \\
\text { uncorrected }\end{array}$ & $\begin{array}{l}\text { Cluster } \\
\text { extent }\end{array}$ & $\begin{array}{c}P_{\text {cluster }} \\
\text { FDR-corrected }\end{array}$ & $\begin{array}{c}t \\
\text { value }\end{array}$ \\
\hline \multicolumn{7}{|l|}{ Capsulotomy } \\
\hline \multirow[t]{5}{*}{ Decreased } & $-8,12,-2$ & Left caudate & $<0.001$ & 6,629 & $<0.001$ & 26.68 \\
\hline & $-4,44,14$ & Left ACC & $<0.001$ & 3,665 & $<0.001$ & 13.00 \\
\hline & $40,-82,-42$ & Right posterior cerebellum & $<0.001$ & 920 & $<0.001$ & 6.44 \\
\hline & $-14,-82,-44$ & Left posterior cerebellum & $<0.001$ & 594 & $<0.001$ & 6.08 \\
\hline & $32,34,-12$ & Right inferior frontal & $<0.001$ & 220 & 0.022 & 6.05 \\
\hline \multirow[t]{6}{*}{ Increased } & $20,-96,-20$ & Right occipital, fusiform/lingual gyrus & $<0.001$ & 1,133 & $<0.001$ & 8.72 \\
\hline & $0,-72,-8$ & Left cerebellum, anterior lobe & $<0.001$ & 683 & $<0.001$ & 7.24 \\
\hline & $-8,-54,72$ & Left parietal, postcentral gyrus + precuneus & $<0.001$ & 861 & $<0.001$ & 6.80 \\
\hline & $42,-78,32$ & Right temporal, angular gyrus + precuneus & $<0.001$ & 224 & 0.040 & 5.65 \\
\hline & $52,-24,38$ & Right parietal, postcentral gyrus & $<0.001$ & 240 & 0.040 & 5.09 \\
\hline & $62,-44,-8$ & Right middle temporal & $<0.001$ & 215 & 0.040 & 4.76 \\
\hline \multicolumn{7}{|l|}{ DBS } \\
\hline \multirow[t]{4}{*}{ Decreased } & $-30,22,-16$ & Left inferior frontal & $<0.001$ & 1,491 & $<0.001$ & 8.81 \\
\hline & $-20,62,-10$ & Left superior frontal & $<0.001$ & 640 & 0.001 & 8.52 \\
\hline & $4,36,-10$ & Right ACC & $<0.001$ & 2,213 & $<0.001$ & 6.29 \\
\hline & $36,42,-14$ & Right middle frontal & $<0.001$ & 2,584 & $<0.001$ & 6.09 \\
\hline
\end{tabular}

(lingual gyrus). No significant correlation was found for the change in HAM-D score in either group.

Preoperative metabolism in the DBS group and changes in YBOCS scores did not significantly correlate with an uncorrected $P_{\text {height }}$ of less than 0.001 . However, when we relaxed significance levels down to an uncorrected $P_{\text {height }}$ of less than 0.01 and an FDRcorrected $P_{\text {cluster }}$ of more than 0.05 , we did find a negative correlation between changes in Y-BOCS scores and the preoperative metabolism in the pregenual and dorsal anterior cingulate (only without age and sex as nuisance variables), consistent with our previous pilot study (11).

In the DBS group, there was a significant negative correlation between change in HAM-D score and preoperative metabolism in the left superior temporal gyrus, and there was a trend toward significance (uncorrected $P_{\text {height }}<0.005$ and FDR-corrected $P_{\text {cluster }}=$ 0.066 , only without age and sex as nuisance variables) in the pregenual and dorsal anterior cingulate (Supplemental Fig. 1; Supplemental Table 1 [available at http://jnm.snmjournals.org]).

\section{DISCUSSION}

Current neurosurgical procedures for psychiatric disorders such as OCD are based on strong evidence of central involvement of the CSPTC circuit. The most important CSPTC loop involved in OCD is the limbic loop, consisting of the medial orbitofrontal cortex and the anterior cingulate, projecting to the mediodorsal thalamus.

Previous studies have described an increased baseline metabolism in some CSPTC structures in OCD patients (9). Our study confirmed the baseline findings of increased metabolism in the anterior cingulate. There was also an increased baseline metabolism in the occipital cortex (cuneus) and the posterior cerebellum (semilunar lobule). This finding is in line with previous studies reporting these other brain regions outside the CSPTC to be important in OCD symptomatology $(20,21)$.

Furthermore, we found reduced metabolism in the posterior cingulate cortex, the superior frontal cortex, the supplementary motor cortex, and the inferior parietal cortex, again in agreement with previous findings $(11,22,23)$. In particular, Saxena et al. showed an elevated frontal metabolism at baseline in patients with comorbid OCD and major depressive disorder (MD) (22). In MD, several cognitive and motor deficits have been explained by neuronal dysfunction in parts of the frontal cortex, as well as in the parietal cortex and premotor cortex $(24,25)$. Because our cohort consisted mainly of refractory OCD patients with depression, such comorbidity can explain our findings and, thus, anatomic or functional differences from patients with MD or OCD alone can be present.

Significant decreases in postoperative metabolism were found in parts of the frontal cortex and anterior cingulate, as well as in the medial dorsal thalamus, the left caudate nucleus, and parts of the posterior cerebellum.

With respect to the subgenual ACC, our analyses showed a significant postoperative decrease in both the capsulotomy group and the DBS group, confirming the findings of our previous pilot study (11). Zuo et al. also showed a metabolic decrease in the ACC after capsulotomy in OCD patients (12). The anterior cingulate has been described as an important region in both OCD and MD symptomatology. Mayberg found a hypermetabolic state in the subgenual cingulate cortex in depressed patients and a decrease

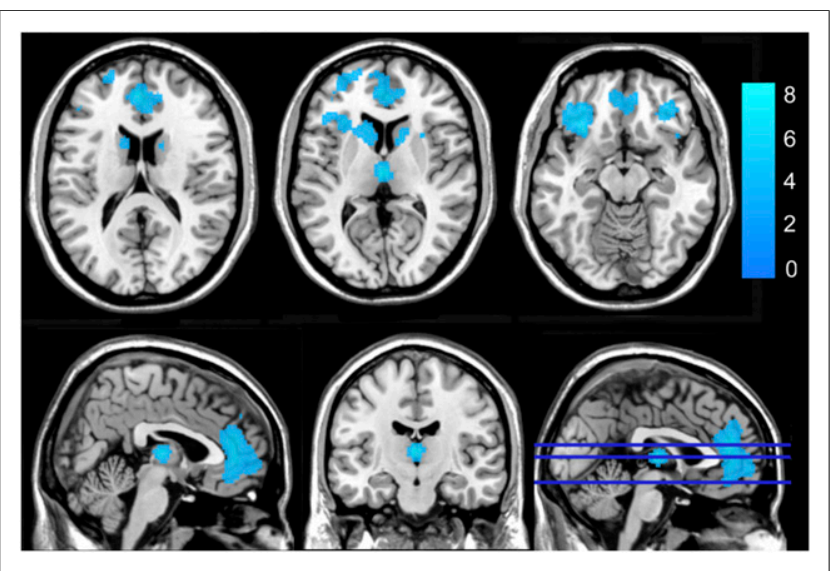

FIGURE 4. Clusters of joint decreased changes in metabolic activity in capsulotomy and DBS groups after conjunction analysis with uncorrected $P_{\text {height }}<0.001$ and FDR-corrected $P_{\text {cluster }}<0.05$. 
TABLE 5

Coordinates and Statistics of Cluster Peaks of Jointly Significant Postoperative Decreases in Metabolic Activity in CAPS and DBS Groups After Conjunction Analysis

\begin{tabular}{|c|c|c|c|c|c|}
\hline$x, y, z(\mathrm{~mm})$ & Brain area & $P_{\text {height }}$, uncorrected & Cluster extent & $P_{\text {cluster }}$, FDR-corrected & $t$ value \\
\hline$-32,24,-8$ & Left inferior frontal & $<0.001$ & 2,233 & $<0.001$ & 6.82 \\
\hline$-2,44,18$ & ACC & $<0.001$ & 1,844 & $<0.001$ & 6.48 \\
\hline $0,-16,6$ & MD thalamus & $<0.001$ & 214 & 0.103 & 6.86 \\
\hline
\end{tabular}

after successful pharmacologic treatment (26). Such decreased metabolism was also present after successful treatment by chronic DBS in major depression (27). Of note, multiple studies have reported a decreased metabolism in this region after successful pharmacologic treatment of both OCD and MD (27-29).

In parts of the prefrontal (left inferior and superior frontal and right middle frontal) and orbitofrontal cortices, there were significant decreases in the DBS-on group, compared with both the preoperative and the DBS-off conditions. There was no overlap with the frontal regions of decreased metabolism at baseline. Chronic successful treatment has been associated with a decrease in prefrontal and orbitofrontal metabolism in several studies $(12,30,31)$. This decrease correlated with improvement of OCD symptoms in several studies $(8,30)$. Although we could not confirm an aberrant baseline metabolism, in this study group the results showed DBS to have an important effect on metabolism in this region.

A significant metabolic decrease was seen in the medial dorsal thalamus and the caudate head. Both regions have been extensively documented to be core structures in the CSPTC. Zuo et al. showed a metabolic decrease in these structures after capsulotomy in OCD patients (12), and a trend toward a metabolic decrease in the MD thalamus was shown in a previous pilot study after DBS (11). Other studies have also noted decreased activity in these regions after successful pharmacologic or behavioral treatment of $\operatorname{OCD}(28,30,31)$, emphasizing the importance of these regions in treatment response of OCD.

Regarding the cerebellum, our results showed decreased metabolism in the pyramis after capsulotomy. Previous studies reported involvement of the cerebellum in OCD symptomatology $(23,30,32)$. Zuo et al. (12) showed a postoperative decrease in the cerebellar tonsil, inferior to the pyramis. In their study there was also a correlation of baseline metabolism in the cerebellar tonsil with clinical improvement after capsulotomy. The cerebellum has been associated with multiple cognitive functions such as attention, memory, and learning, which are often affected in OCD patients.

Regional metabolism in the parietal cortex and the precuneus was relatively increased postoperatively. This finding is at odds

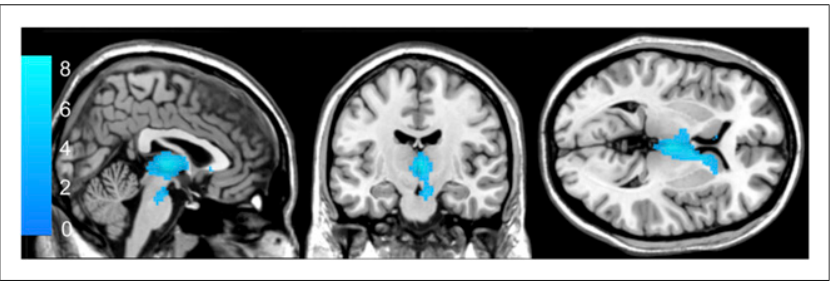

FIGURE 5. Comparison of metabolic changes after capsulotomy vs. DBS. Results are for complete patient groups. Decrease in metabolism in medial dorsal thalamus and caudate heads was more pronounced after capsulotomy. with previous work on OCD in which the precuneus was hypermetabolic after symptom provocation tests $(20,23,33)$, and a trend toward a positive correlation between baseline metabolism and symptom severity has been shown (34). The precuneus is part of the default network (21). A dissociation of functions inside the default network has been described (35). The default network seems to be a heterogeneous system with at least 2 distinct subsystems: the medial temporal lobe and the dorsal medial prefrontal cortex. The precuneus is part of the medial temporal lobe subsystem, which seems to be involved in making decisions about one's future. In contrast, the dorsal medial prefrontal cortex subsystem is more active when considering one's own present mental state. Although speculative, the existence of functional anatomic subsystems inside the default network may explain why results from multiple studies differ.

An increased metabolism after capsulotomy was demonstrated in the right occipital cortex (fusiform and lingual gyrus). Zuo et al. (12) also reported an increased postoperative metabolism in this region.

For the medial dorsal thalamus and the caudate heads, a significantly larger decrease in activity was found in the capsulotomy group than in the DBS group. The ablative character of the intervention may explain this finding. During capsulotomy, the lesion that is made in the anterior limb of the internal capsule may destroy projections to the medial dorsal thalamus. In DBS, the structural lesion caused by implanting the electrode is less elaborate. This idea is further supported by the fact that there was no significant metabolic difference between the DBS-off scans and the preoperative scans for DBS, unless we relaxed the prespecified significance levels. Moreover, a larger and more extended metabolic change in the capsulotomy group than in the DBS group was found. Although no formal statistical correlation analysis was possible because of the small numbers, this finding may be related to the more pronounced side effects of frontal lobe dysfunction observed in the capsulotomy groups (i.e., apathy, emotional blunting, and disinhibition). In the DBS group, none of these side effects were seen.

Regarding the relationship to clinical scores, in earlier reports the subgenual ACC was mentioned as an important structure in OCD and depression symptomatology. Dougherty et al. (36) and Zuo et al. (12) showed a correlation between preoperative metabolism at this site and improvement in, respectively, depressive symptoms and OCD symptoms after ablative surgery. In our pilot study (11), preoperative metabolism in the subgenual ACC was predictive of improvement in postoperative Y-BOCS scores in a population of 6 OCD patients with comorbid depressive symptoms. This was also the case for the DBS group in the current study, but only when significance levels were relaxed. These data highlight the subgenual cingulate as an important cross-point for pathophysiology and treatment effects in OCD.

At this stage, the role of the occipital cortex in OCD symptomatology is not well understood. In the cuneus and lingual gyrus for the capsulotomy group, we found a negative correlation between 
TABLE 6

Coordinates and Statistics of Significant Differences in Metabolic Decreases Between CAPS and DBS Groups

\begin{tabular}{llcccc}
\hline$x, y, z(\mathrm{~mm})$ & \multicolumn{1}{c}{ Brain area } & $P_{\text {height }}$, uncorrected & Cluster extent & $P_{\text {cluster }}$, FDR-corrected & $t$ value \\
\hline $2,-14,2$ & Right mediodorsal thalamus & $<0.001$ & 1,350 & $<0.001$ & 7.02 \\
$-6,14,-8$ & Left caudate head & $<0.001$ & 177 & 0.28 & 4.37 \\
\hline
\end{tabular}

OCD symptomatology and the change in Y-BOCS scores (i.e., better outcome with increased postoperative metabolism). Bussato et al. (32) described an improvement in Y-BOCS score to be related to an increase in perfusion in the left cuneus. In patients after capsulotomy, increases in the right middle occipital gyrus related to Y-BOCS improvement (12). Another study showed metabolic increases in the cuneus after successful pharmacologic treatment of OCD (36).

A negative correlation was also found between postoperative HAM-D changes and preoperative metabolism in the superior temporal cortex. Previous studies already suggested that the superior temporal cortex has a role in OCD with comorbid MD (29), as well as in OCD (23) or MD alone (24).

Several limitations need to be addressed. In our study design, patients were not randomized between capsulotomy and DBS. The stricter criteria for DBS than for capsulotomy may have led to some selection bias, as patients selected for DBS needed to be more functionally competent to ascertain a complete follow-up. However, bias was not reflected in differences in their Y-BOCS or HAM-D scores or changes therein.

Second, the site of stimulation evolved during the study to a more posterior position in the DBS group but remained in the ventral striatum below the anterior limb of the anterior capsule, as retrospectively confirmed by postoperative MR imaging. It is conceivable that the variation of the implantation site can have an impact on the neural circuits highlighted in the PET study.

Third, the male-female distribution in the capsulotomy and DBS groups was significantly different from that in our healthy control group, but since we predominantly investigated within-subject differences, this difference can be seen as only a second-order effect.

Furthermore, between-subject differences in medication existed, and it is known that selective reuptake inhibitors may induce metabolic decreases in the ACC, middle temporal cortex, precuneus, and parahippocampal region (37). However, since medication was kept low and stable during the imaging period, medication is unlikely to have been a confounder in the current results.

Last, data were analyzed using proportional scaling, which neglects potential differences in global metabolism between conditions. However, the assumption of an absence of global effects has been made in most previous studies on OCD, and future studies are needed to address the validity of this hypothesis.

\section{CONCLUSION}

Capsulotomy and DBS result in similar improvements in symptomatology in OCD and result in similar metabolic changes in the CSPTC. However, the changes are more pronounced in capsulotomy than in DBS and extend to areas outside the CSPTC as well. Metabolic activity largely reverses in DBS when stimulation is turned off. Other cortical regions, such as occipital, temporal, and cerebellar structures, may also play an important role in OCD symptomatology. Furthermore, pretreatment metabolism in the superior temporal gyrus and anterior cingulate may predict outcome in OCD patients with comorbid depressive symptoms.

\section{DISCLOSURE}

The costs of publication of this article were defrayed in part by the payment of page charges. Therefore, and solely to indicate this fact, this article is hereby marked "advertisement" in accordance with 18 USC section 1734. Bart Nuttin and Loes Gabriëls hold a chair for neurosurgery for psychiatric disorders, sponsored by Medtronic. Both received funds for research, traveling, and teaching from Medtronic. Bart Nuttin authors a patent on DBS for OCD. Medtronic provided the implants and replacements of the implants for the DBS patients for free until the moment of official reimbursement of those devices in Belgium. Koen Van Laere is senior clinical investigator for the Flemish Fund for Scientific Research (FWO, Fonds voor Wetenschappelijk Onderzoek Vlaanderen). Financial support was provided by the FWO (project G.0729.09N) and the Agency for Innovation by Science and Technology (IWT 100683). No other potential conflict of interest relevant to this article was reported.

\section{REFERENCES}

1. Ruscio AM, Stein DJ, Chiu WT, Kessler RC. The epidemiology of obsessivecompulsive disorder in the National Comorbidity Survey Replication. Mol Psychiatry. 2010;15:53-63.

2. Jenike MA. Neurosurgical treatment of obsessive-compulsive disorder. $\mathrm{Br} J$ Psychiatry. 1998;(35):79-90.

3. Nuttin B, Cosyns P, Demeulemeester H, Gybels J, Meyerson B. Electrical stimulation in anterior limbs of internal capsules in patients with obsessive-compulsive disorder. Lancet. 1999;354:1526.

4. Greenberg BD, Gabriëls LA, Malone DA Jr, et al. Deep brain stimulation of the ventral internal capsule/ventral striatum for obsessive-compulsive disorder: worldwide experience. Mol Psychiatry. 2010;15:64-79.

5. Rauch SL. Neuroimaging and neurocircuitry models pertaining to the neurosurgical treatment of psychiatric disorders. Neurosurg Clin N Am. 2003;14:213-223.

6. Kopell BH, Greenberg BD. Anatomy and physiology of the basal ganglia: implications for DBS in psychiatry. Neurosci Biobehav Rev. 2008;32:408-422.

7. Saxena S, Brody AL, Schwartz JM, Baxter LR. Neuroimaging and frontal-subcortical circuitry in obsessive-compulsive disorder. Br J Psychiatry Suppl. 1998;(35):26-37.

8. Le Jeune F, Verin M, N'Diaye K, et al. Decrease of prefrontal metabolism after subthalamic stimulation in obsessive-compulsive disorder: a positron emission tomography study. Biol Psychiatry. 2010;68:1016-1022.

9. Saxena S, Brody AL, Maidment KM, et al. Cerebral glucose metabolism in obsessive-compulsive hoarding. Am J Psychiatry. 2004;161:1038-1048.

10. Rauch SL, Dougherty DD, Malone D, et al. A functional neuroimaging investigation of deep brain stimulation in patients with obsessive-compulsive disorder. J Neurosurg. 2006;104:558-565.

11. Van Laere K, Nuttin B, Gabriëls L, et al. Metabolic imaging of anterior capsular stimulation in refractory obsessive-compulsive disorder: a key role for the subgenual anterior cingulate and ventral striatum. J Nucl Med. 2006;47:740-747.

12. Zuo C, Ma Y, Sun B, et al. Metabolic imaging of bilateral anterior capsulotomy in refractory obsessive compulsive disorder: an FDG PET study. J Cereb Blood Flow Metab. 2013;33:880-887.

13. Diagnostic and Statistical Manual of Mental Disorders. 4th ed., text revision. Washington, DC: American Psychiatry Association; 2000.

14. Gabriëls L, Nuttin B, Cosyns P. Applicants for stereotactic neurosurgery for psychiatric disorders: role of the Flemish advisory board. Acta Psychiatr Scand. 2008; 117:381-389.

15. Goodman WK, Price LH, Rasmussen SA, et al. The Yale-Brown Obsessive Compulsive Scale. I. Development, use, and reliability. Arch Gen Psychiatry. 1989;46:1006-1011.

16. Hamilton M. A rating scale for depression. J Neurol Neurosurg Psychiatry. 1960;23:56-62. 
17. Nuttin B, Gielen F, van Kuyck K, et al. Targeting bed nucleus of the stria terminalis for severe obsessive-compulsive disorder: more unexpected lead placement in obsessive-compulsive disorder than in surgery for movement disorders. World Neurosurg. 2013;80:S30 e11-S30 e16.

18. Asanuma K, Tang C, Ma Y, et al. Network modulation in the treatment of Parkinson's disease. Brain. 2006;129:2667-2678.

19. Lancaster JL, Woldorff MG, Parsons LM, et al. Automated Talairach atlas labels for functional brain mapping. Hum Brain Mapp. 2000;10:120-131.

20. Mataix-Cols D, Wooderson S, Lawrence N, Brammer MJ, Speckens A, Phillips ML. Distinct neural correlates of washing, checking, and hoarding symptom dimensions in obsessive-compulsive disorder. Arch Gen Psychiatry. 2004;61:564-576.

21. Raichle ME, MacLeod AM, Snyder AZ, Powers WJ, Gusnard DA, Shulman GL. A default mode of brain function. Proc Natl Acad Sci USA. 2001;98:676-682.

22. Saxena S, Brody AL, Ho ML, et al. Cerebral metabolism in major depression and obsessive-compulsive disorder occurring separately and concurrently. Biol Psychiatry. 2001;50:159-170.

23. Sanematsu H, Nakao T, Yoshiura T, et al. Predictors of treatment response to fluvoxamine in obsessive-compulsive disorder: an fMRI study. $J$ Psychiatr Res. 2010;44:193-200.

24. Videbech P. PET measurements of brain glucose metabolism and blood flow in major depressive disorder: a critical review. Acta Psychiatr Scand. 2000;101:11-20.

25. Bench CJ, Friston KJ, Brown RG, Frackowiak RS, Dolan RJ. Regional cerebral blood flow in depression measured by positron emission tomography: the relationship with clinical dimensions. Psychol Med. 1993;23:579-590.

26. Mayberg HS. Limbic-cortical dysregulation: a proposed model of depression. J Neuropsychiatry Clin Neurosci. 1997;9:471-481.

27. Mayberg HS, Lozano AM, Voon V, et al. Deep brain stimulation for treatmentresistant depression. Neuron. 2005;45:651-660.
28. Perani D, Colombo C, Bressi S, et al. $\left[{ }^{18} \mathrm{~F}\right] \mathrm{FDG}$ PET study in obsessive-compulsive disorder: a clinical/metabolic correlation study after treatment. $\mathrm{Br} J$ Psychiatry. 1995; 166:244-250.

29. Saxena S, Brody AL, Ho ML, et al. Differential cerebral metabolic changes with paroxetine treatment of obsessive-compulsive disorder vs major depression. Arch Gen Psychiatry. 2002;59:250-261.

30. Swedo SE, Pietrini P, Leonard HL, et al. Cerebral glucose metabolism in childhood-onset obsessive-compulsive disorder: revisualization during pharmacotherapy. Arch Gen Psychiatry. 1992;49:690-694.

31. Saxena S, Brody AL, Maidment KM, et al. Localized orbitofrontal and subcortical metabolic changes and predictors of response to paroxetine treatment in obsessive-compulsive disorder. Neuropsychopharmacology. 1999;21:683-693.

32. Busatto GF, Zamignani DR, Buchpiguel CA, et al. A voxel-based investigation of regional cerebral blood flow abnormalities in obsessive-compulsive disorder using single photon emission computed tomography (SPECT). Psychiatry Res. 2000;99:15-27.

33. Cavanna AE, Trimble MR. The precuneus: a review of its functional anatomy and behavioural correlates. Brain. 2006;129:564-583.

34. McGuire PK, Bench CJ, Frith CD, Marks IM, Frackowiak RS, Dolan RJ. Functional anatomy of obsessive-compulsive phenomena. Br J Psychiatry. 1994;164:459-468.

35. Broyd SJ, Demanuele C, Debener S, Helps SK, James CJ, Sonuga-Barke EJ. Default-mode brain dysfunction in mental disorders: a systematic review. Neurosci Biobehav Rev. 2009;33:279-296.

36. Dougherty DD, Weiss AP, Cosgrove GR, et al. Cerebral metabolic correlates as potential predictors of response to anterior cingulotomy for treatment of major depression. J Neurosurg. 2003;99:1010-1017.

37. Smith GS, Kramer E, Hermann C, et al. Serotonin modulation of cerebral glucose metabolism in depressed older adults. Biol Psychiatry. 2009;66:259-266. 